PROCEEDINGS OF THE

AMERICAN MATHEMATICAL SOCIETY

Volume 140, Number 4, April 2012, Pages 1133-1139

S 0002-9939(2011)10942-0

Article electronically published on November 23, 2011

\title{
HOCHSCHILD HOMOLOGY AND TRUNCATED CYCLES
}

\author{
PETTER ANDREAS BERGH, YANG HAN, AND DAG MADSEN
}

(Communicated by Birge Huisgen-Zimmermann)

Dedicated to Professor Claus Michael Ringel on the occasion of his 65th birthday

\begin{abstract}
We study algebras having 2-truncated cycles and show that these algebras have infinitely many nonzero Hochschild homology groups. Consequently, algebras of finite global dimension have no 2-truncated cycles and therefore satisfy a higher version of the "no loops conjecture".
\end{abstract}

\section{INTRODUCTION}

In his 1989 paper Hap, Happel noted that if a finite dimensional algebra over an algebraically closed field is of finite global dimension, then all its higher Hochschild cohomology groups vanish. He then remarked that the converse was unknown, and this gave birth to "Happel's question". Namely, if all the higher Hochschild cohomology groups vanish for a finite dimensional algebra (not necessarily over an algebraically closed ground field), then must the algebra have finite global dimension? A positive answer for commutative algebras was provided in AvI. However, it was shown in BGMS that this does not hold in general.

The homology version of Happel's question, first conjectured by the second author in $\mathrm{Han}$, is open: if all the higher Hochschild homology groups of a finite dimensional algebra vanish, then is the algebra of finite global dimension? As in the cohomology case, the answer is yes for commutative algebras (cf. [AV-P]). Moreover, the conjecture has been established for large classes of noncommutative algebras in BM1, [BM2, [Han] and [SV-P, for example monomial algebras, Koszul algebras, cellular algebras and local graded algebras.

In this paper, we focus on the combinatorics of quivers of algebras. We show that the conjecture holds for all algebras having 2-truncated cycles, that is, cycles in which the product of two consecutive arrows is always zero. In other words, we show that such algebras have infinitely many nonzero Hochschild homology groups. Consequently, a higher version of the well-known "no loops conjecture" holds: algebras of finite global dimension have no 2-truncated cycles.

We thank the referee for valuable suggestions, especially on the proof of the main theorem.

Received by the editors July 12, 2010 and, in revised form, October 27, 2010 and November 26, 2010 .

2010 Mathematics Subject Classification. Primary 16E40.

Key words and phrases. Hochschild homology, truncated cycles.

The first author was supported by NFR Storforsk grant No. 167130.

The second author was supported by Project 10731070 NSFC.

(C)2011 American Mathematical Society Reverts to public domain 28 years from publication 


\section{HochSCHILD HOMOLOGY}

Let $k$ be a commutative ring and $A$ a $k$-algebra. Thus, there exists a nonzero ring homomorphism $k \rightarrow A$, whose image is contained in the center of $A$. We do not assume that $A$ is finitely generated as a $k$-module.

For each $n \geq 1$, denote by $A^{\otimes n}$ the $n$-fold tensor product $A \otimes_{k} \cdots \otimes_{k} A$, in which there are $n$ copies of $A$. Moreover, consider the map $A^{\otimes(n+1)} \stackrel{d_{n}}{\longrightarrow} A^{\otimes n}$ given by

$$
\begin{aligned}
a_{0} \otimes \cdots \otimes a_{n} \mapsto & \sum_{i=0}^{n-1}(-1)^{i} a_{0} \otimes \cdots \otimes a_{i} a_{i+1} \otimes \cdots \otimes a_{n} \\
& +(-1)^{n} a_{n} a_{0} \otimes a_{1} \otimes \cdots \otimes a_{n-1} .
\end{aligned}
$$

Then $d_{n} d_{n+1}=0$, and so we may form a complex

$$
\cdots \rightarrow A^{\otimes 4} \stackrel{d_{3}}{\longrightarrow} A^{\otimes 3} \stackrel{d_{2}}{\longrightarrow} A^{\otimes 2} \stackrel{d_{1}}{\longrightarrow} A,
$$

in which $A^{\otimes n}$ is of degree $n-1$. The $n$th homology group of this complex, denoted $\mathrm{HH}_{n}(A)$, is the $n$th Hochschild homology group of our algebra $A$. Thus $\mathrm{HH}_{n}(A)$ is the abelian group

$$
\operatorname{Ker}\left(A^{\otimes(n+1)} \stackrel{d_{n}}{\longrightarrow} A^{\otimes n}\right) / \operatorname{Im}\left(A^{\otimes(n+2)} \stackrel{d_{n+1}}{\longrightarrow} A^{\otimes(n+1)}\right),
$$

where $d_{0}$ is the zero map. This is a $k$-module, in general not finitely generated since $A$ is not assumed to be module finite over $k$. We refer to the above complex as the Hochschild complex of $A$. The main emphasis of this paper is on algebras having infinitely many nonzero Hochschild homology groups. We therefore define

$$
\operatorname{HHdim} A \stackrel{\text { def }}{=} \sup \left\{n \in \mathbb{Z} \mid \mathrm{HH}_{n}(A) \neq 0\right\} .
$$

This is the Hochschild homology dimension of the algebra $A$.

Hochschild homology (unlike Hochschild cohomology) is functorial in the category of $k$-algebras. Namely, let $A$ and $B$ be two $k$-algebras and suppose that $A \stackrel{f}{\rightarrow} B$ is a $k$-algebra homomorphism. Then $f$ is a homomorphism of rings, and there is a commutative diagram

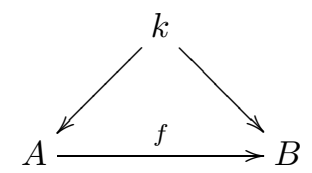

of maps, where $k \rightarrow A$ and $k \rightarrow B$ are the maps giving the $k$-algebra structure of $A$ and $B$. This implies that for each $n \geq 1$, the diagram

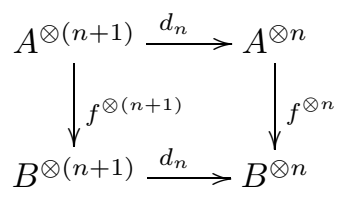

commutes, giving a map

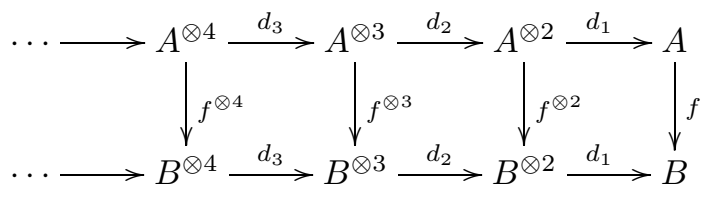


of Hochschild complexes. For each $n \geq 0$, this map of Hochshild complexes induces a map $\mathrm{HH}_{n}(A) \stackrel{f^{\otimes(n+1)}}{\longrightarrow} \mathrm{HH}_{n}(B)$ of Hochschild homology groups, given by

$$
\sum_{i} a_{0}^{i} \otimes \cdots \otimes a_{n}^{i}+\operatorname{Im} d_{n+1} \mapsto \sum_{i} f\left(a_{0}^{i}\right) \otimes \cdots \otimes f\left(a_{n}^{i}\right)+\operatorname{Im} d_{n+1} .
$$

This simple fact is the key ingredient in the proof of the main theorem, where we want to show that a certain Hochschild homology group, say $\mathrm{HH}_{n}(A)$, is nonzero. Namely, we construct an algebra homomorphism $A \rightarrow B$, where $B$ is an algebra which has Hochschild homology groups that are more amenable to computation. If we can show that the image of $\mathrm{HH}_{n}(A) \rightarrow \mathrm{HH}_{n}(B)$ is nonzero, then this forces $\mathrm{HH}_{n}(A)$ to be nonzero.

\section{Truncated CyCles}

In this final section we prove the main result, which shows that certain algebras have nonzero Hochschild homology groups in arbitrarily high degrees. We formulate this result for path algebras of quivers. Recall that if $k$ is a commutative ring and $Q$ a quiver with a finite number of vertices $\{1, \ldots, n\}$, then the path algebra $k Q$ is the unital $k$-algebra defined as follows. As a $k$-module, the algebra $k Q$ is free with basis $e_{1}, \ldots, e_{n}$ and all possible finite sequences

$$
i_{1} \stackrel{x_{1}}{\longrightarrow} i_{2} \stackrel{x_{2}}{\longrightarrow} i_{3} \rightarrow \cdots \rightarrow i_{w} \stackrel{x_{w}}{\longrightarrow} i_{w+1}
$$

of arrows. We write the latter as $x_{w} \cdots x_{1}$, denote its initial vertex $i_{1}$ by $s\left(x_{w} \cdots x_{1}\right)$, and its terminal vertex $i_{w+1}$ by $t\left(x_{w} \cdots x_{1}\right)$. Moreover, we view each $e_{i}$ as a trivial path, with $s\left(e_{i}\right)=i=t\left(e_{i}\right)$. If $p$ is any path and $1 \leq i \leq n$, then multiplication of $p$ and $e_{i}$ is defined as

$$
p \cdot e_{i}=\left\{\begin{array}{ll}
p & \text { if } s(p)=i \\
0 & \text { if } s(p) \neq i,
\end{array} \quad e_{i} \cdot p= \begin{cases}p & \text { if } t(p)=i \\
0 & \text { if } t(p) \neq i .\end{cases}\right.
$$

Finally, if $p$ and $q$ are nontrivial paths, then their product is defined as

$$
p \cdot q= \begin{cases}p q & \text { if } s(p)=t(q) \\ 0 & \text { if } s(p) \neq t(q),\end{cases}
$$

i.e. the concatenation of the two paths when possible. Thus the unit in $k Q$ is $e_{1}+\cdots+e_{n}$, and the elements $e_{1}, \ldots, e_{n}$ are orthogonal idempotents. We denote the two-sided ideal generated by the arrows by $J_{k Q}$.

The importance of path algebras is established in a classical result of Gabriel. Recall that an ideal $I$ in a path algebra $k Q$ is admissible if $J_{k Q}^{n} \subseteq I \subseteq J_{k Q}^{2}$ for some $n \geq 2$. Gabriel showed that every finite dimensional basic algebra over an algebraically closed field $k$ is isomorphic to a factor $k Q / I$ of a path algebra, where $Q$ is some finite quiver and $I \subseteq k Q$ is an admissible ideal (cf. [ASS, Theorem II.3.7]). Moreover, every finite dimensional algebra over a field is Morita equivalent to a finite dimensional basic algebra over the same field.

If $I \subseteq J_{k Q}^{2}$ is an ideal in the path algebra $k Q$, then a cycle in $k Q / I$ is a finite sequence $x_{1}, \ldots, x_{u}$ of arrows with the property that $t\left(x_{i}\right)=s\left(x_{i+1}\right)$ and $t\left(x_{u}\right)=$ $s\left(x_{1}\right)$. Every algebra $k Q / I$ of infinite global dimension over a field contains a cycle; this follows from [ENN, Theorem 8]. 
Definition. A cycle $x_{1}, \ldots, x_{u}$ in $k Q / I$ is $m$-truncated (for an integer $m \geq 2$ ) if

$$
x_{i+m-1} \cdots x_{i+1} x_{i}=0 \quad \text { and } \quad x_{i+m-2} \cdots x_{i+1} x_{i} \neq 0
$$

for all $i$ (where the indices are modulo $u$ ).

The main result deals with path algebras whose quivers contain 2-truncated cycles, that is, cycles in which the product of any two consecutive arrows is zero. Note that the algebras considered are not necessarily finitely generated over the commutative ground ring: although we assume the quiver has finitely many vertices, there is no assumption on the number of arrows. In the special case when the algebra is local, the theorem provides a generalization of [SV-P, Theorem II].

Theorem 3.1. Let $k$ be a commutative ring, $Q$ a quiver with a finite number of vertices, and $I \subseteq k Q$ an ideal contained in $J_{k Q}^{2}$. Suppose $k Q / I$ contains a 2 truncated cycle $x_{1}, \ldots, x_{u}$. Then for every $n \geq 1$ with un $\equiv u(\bmod 2)$, the element

$$
\left(x_{u} \otimes \cdots \otimes x_{1}\right)^{\otimes n}
$$

represents a nonzero element in $\mathrm{HH}_{u n-1}(k Q / I)$. In particular, $\operatorname{HHdim} k Q / I=\infty$.

Proof. We denote the algebra $k Q / I$ by $A$. We may assume that the 2 -truncated cycle is minimal, i.e. that $x_{i} \neq x_{j}$ whenever $i \neq j$ : if not, there is a smaller 2-truncated cycle.

Consider the Hochschild homology complex

$$
\cdots \rightarrow A^{\otimes(u n+1)} \stackrel{d_{u n}}{\longrightarrow} A^{\otimes u n} \stackrel{d_{u n-1}}{\longrightarrow} A^{\otimes(u n-1)} \rightarrow \cdots
$$

and the element $\left(x_{u} \otimes \cdots \otimes x_{1}\right)^{\otimes n}$ in $A^{\otimes u n}$. Since the cycle is 2-truncated, this element belongs to Ker $d_{u n-1}$; hence it represents an element $\xi \in \mathrm{HH}_{u n-1}(A)$. Let $B$ be the algebra $B=k Q / J_{k Q}^{2}$, and consider the natural surjective $k$-algebra homomorphism $A \stackrel{f}{\rightarrow} B$. The image of $\xi$ under the map $\operatorname{HH}_{u n-1}(A) \stackrel{f^{\otimes u n}}{\longrightarrow} \mathrm{HH}_{u n-1}(B)$ is represented by $\left(x_{u} \otimes \cdots \otimes x_{1}\right)^{\otimes n} \in B^{\otimes u n}$. If $\left(x_{u} \otimes \cdots \otimes x_{1}\right)^{\otimes n} \in B^{\otimes u n}$ represents a nonzero element in $\mathrm{HH}_{u n-1}(B)$, then $\left(x_{u} \otimes \cdots \otimes x_{1}\right)^{\otimes n} \in A^{\otimes u n}$ represents a nonzero element in $\mathrm{HH}_{u n-1}(A)$.

The algebra $B$ has a grading $B=B_{0} \oplus B_{1}$ with all arrows having degree one. The degree un layer of the Hochschild complex has the following shape:

$$
\cdots \rightarrow\left(B^{\otimes u n+1}\right)_{\text {un }} \stackrel{d_{u n}}{\longrightarrow}\left(B^{\otimes u n}\right)_{\text {un }} \stackrel{d_{u n-1}}{\longrightarrow} 0 \rightarrow \cdots .
$$

The $k$-module, $\left(B^{\otimes u n}\right)_{u n}$ is generated by elements of the form

$$
z_{1} \otimes \cdots \otimes z_{u n},
$$

where each $z_{j}$ is an arrow. As a $k$-module, $\left(B^{\otimes u n}\right)_{u n}$ decomposes as

$$
\left(B^{\otimes u n}\right)_{u n}=W \oplus U,
$$

where $W$ is the submodule of $\left(B^{\otimes u n}\right)_{u n}$ generated by those generators $z_{1} \otimes \cdots \otimes z_{u n}$ having the property that the path $z_{1} \cdots z_{u n}$ is a cyclic shift of the path $\left(x_{u} \cdots x_{1}\right)^{n}$ in $k Q$, and $U$ is the submodule generated by all remaining generators. Similarly, the $k$-module $\left(B^{\otimes u n+1}\right)_{u n}$ is generated by elements of the form $z_{0} \otimes \cdots \otimes z_{u n}$, where exactly one $z_{j}$ is a trivial path and all other $z_{j}$ are arrows. As a $k$-module, $\left(B^{\otimes u n+1}\right)_{u n}$ decomposes as

$$
\left(B^{\otimes u n+1}\right)_{u n}=W^{\prime} \oplus U^{\prime}
$$


where $W^{\prime}$ is the submodule of $\left(B^{\otimes u n+1}\right)$ un generated by those generators $z_{0} \otimes$ $\cdots \otimes z_{u n}$ having the property that the path $z_{0} \cdots z_{u n}$ is a cyclic shift of the path $\left(x_{u} \cdots x_{1}\right)^{n}$ in $k Q$, and $U^{\prime}$ is the submodule generated by all remaining generators. We have $d_{u n}\left(W^{\prime}\right) \subseteq W$ and $d_{u n}\left(U^{\prime}\right) \subseteq U$.

We must show that $\left(x_{u} \otimes \cdots \otimes x_{1}\right)^{\otimes n} \in B^{\otimes u n}$ does not belong to Im $d_{u n}$. Suppose therefore that there exists an element $w \in B^{\otimes(u n+1)}$ with the property that $\left(x_{u} \otimes\right.$ $\left.\cdots \otimes x_{1}\right)^{\otimes n}=d_{u n}(w)$. Since

$$
\left(x_{u} \otimes \cdots \otimes x_{1}\right)^{\otimes n} \in W \subseteq\left(B^{\otimes u n}\right)_{u n},
$$

we may assume that

$$
w \in W^{\prime} \subseteq\left(B^{\otimes u n+1}\right)_{\text {un }} .
$$

Thus we can write $w$ as a $k$-linear combination of elements of the form $z_{0} \otimes \cdots \otimes z_{u n}$, in which exactly one $z_{j}$ is a trivial path and all other $z_{j}$ are arrows, and $z_{0} \cdots z_{u n}$ is a cyclic shift of $\left(x_{u} \cdots x_{1}\right)^{n}$. In each term we may assume that $z_{0}$ is the trivial path, since $d_{u n}\left(z_{0} \otimes \cdots \otimes z_{u n}\right)=0$ in all other cases.

From what we have shown so far, we may assume that $w$ can be written as

$$
\begin{gathered}
w=\quad b_{1} \otimes\left(x_{u} \otimes \cdots \otimes x_{1}\right)^{\otimes n} \\
+b_{2} \otimes\left(x_{1} \otimes x_{u} \otimes \cdots \otimes x_{2}\right)^{\otimes n} \\
\vdots \\
+b_{u} \otimes\left(x_{u-1} \otimes \cdots \otimes x_{1} \otimes x_{u}\right)^{\otimes n},
\end{gathered}
$$

where for $1 \leq i \leq u$ we have $b_{i}=\alpha_{i} e_{i}$ for some $\alpha_{i} \in k$. When we apply $d_{u n}$ to $b_{1} \otimes\left(x_{u} \otimes \cdots \otimes x_{1}\right)^{\otimes n}$, the result is just

$$
\alpha_{1}\left[\left(x_{u} \otimes \cdots \otimes x_{1}\right)^{\otimes n}+(-1)^{u n}\left(x_{1} \otimes x_{u} \otimes \cdots \otimes x_{2}\right)^{\otimes n}\right],
$$

and similarly for the other tensor elements in the expression for $w$. This shows that

$$
\begin{aligned}
d_{u n}(w)= & \alpha_{1}\left[\left(x_{u} \otimes \cdots \otimes x_{1}\right)^{\otimes n}+(-1)^{u n}\left(x_{1} \otimes x_{u} \otimes \cdots \otimes x_{2}\right)^{\otimes n}\right] \\
& +\alpha_{2}\left[\left(x_{1} \otimes x_{u} \otimes \cdots \otimes x_{2}\right)^{\otimes n}+(-1)^{u n}\left(x_{2} \otimes x_{1} \otimes x_{u} \otimes \cdots \otimes x_{3}\right)^{\otimes n}\right] \\
& \vdots \\
& +\alpha_{u}\left[\left(x_{u-1} \otimes \cdots \otimes x_{1} \otimes x_{u}\right)^{\otimes n}+(-1)^{u n}\left(x_{u} \otimes \cdots \otimes x_{1}\right)^{\otimes n}\right],
\end{aligned}
$$

where each $\alpha_{i}$ is an element of $k$. The elements $\alpha_{i}$ must satisfy the equations

$$
\begin{aligned}
\alpha_{1}+(-1)^{u n} \alpha_{u} & =1, \\
\alpha_{2}+(-1)^{u n} \alpha_{1} & =0, \\
\alpha_{3}+(-1)^{u n} \alpha_{2} & =0, \\
& \vdots \\
\alpha_{u}+(-1)^{u n} \alpha_{u-1} & =0,
\end{aligned}
$$

but this is impossible when $n$ satisfies $u n \equiv u(\bmod 2)$. Consequently, for such an $n$, the element $\left(x_{u} \otimes \cdots \otimes x_{1}\right)^{\otimes n} \in B^{\otimes u n}$ represents a nonzero element in $\mathrm{HH}_{u n-1}(B)$. It follows that $\left(x_{u} \otimes \cdots \otimes x_{1}\right)^{\otimes n} \in A^{\otimes u n}$ represents a nonzero element in $\operatorname{HH}_{u n-1}(A)$, and this finishes the proof.

From now on we focus on finite dimensional algebras. As mentioned prior to Theorem 3.1, when the ground field is algebraically closed, then every such algebra is Morita equivalent to a factor of a path algebra. We start with algebras whose 
radicals square to zero. The Hochschild homology groups of such algebras arising from quivers were computed in [Skö] and [Cib].

Corollary 3.2. Let $A$ be a finite dimensional algebra over an algebraically closed field. If $A$ has infinite global dimension and its radical squares to zero, then $\operatorname{HH} \operatorname{dim} A=\infty$.

Proof. We may assume that $A$ is a factor of a path algebra of a finite quiver. Since its global dimension is infinite, the quiver has a cycle, which must be 2-truncated since the radical of the algebra squares to zero.

For finite dimensional algebras, or more generally, for algebras which are projective over the commutative ground ring, the Hochschild homology groups are isomorphic to the bimodule homology groups (cf. [CaE, p. 175]). In other words, if $k$ is a field and $A$ is a finite dimensional $k$-algebra, then $\operatorname{HH}_{n}(A) \simeq \operatorname{Tor}_{n}^{A \otimes_{k} A^{\mathrm{op}}}(A, A)$ for every $n$. If moreover $A$ is separable over $k$, then there is an equality gldim $A=$ $\operatorname{pd}_{A \otimes_{k} A^{\text {op }}} A$ (cf. [Hap, p. 110]), and so in this case, $\mathrm{HH}_{n}(A)$ vanishes for large $n$ whenever the global dimension of $A$ is finite. Now let $Q$ be a finite quiver and $I$ an admissible ideal in the path algebra $k Q$. The finite dimensional path algebra $k Q / I$ is then separable over $k$, for any field $k$. We therefore obtain the following corollary to Theorem 3.1 .

Corollary 3.3. Let $k$ be a field, $Q$ a finite quiver and $I$ an admissible ideal in $k Q$. If the algebra $k Q / I$ has finite global dimension, then it contains no 2-truncated cycle.

This can be thought of as a higher version of the well-known "no loops conjecture", which says that $k Q / I$ has no loop whenever the global dimension is finite. This conjecture was proved in Igu for a large class of finite dimensional algebras, including all those for which the ground field is algebraically closed.

It is natural to ask whether Corollary 3.3 still holds if "2-truncated" is replaced by " $m$-truncated": if the algebra $k Q / I$ has finite global dimension, can it contain an $m$-truncated cycle for some $m \geq 2$ ? We end this paper by establishing this for monomial algebras.

Proposition 3.4. Let $k$ be a field, $Q$ a finite quiver and $I$ an admissible ideal in $k Q$ such that $k Q / I$ is a monomial algebra. If the global dimension of $k Q / I$ is finite, then it contains no $m$-truncated cycle for any $m \geq 2$.

Proof. Suppose the algebra does contain an $m$-truncated cycle. Then there exists a truncated basic cycle algebra overlying $k Q / I$, and this is a self-injective Nakayama algebra of infinite global dimension. By IgZ, Proposition 3.2], the algebra $k Q / I$ is also of infinite global dimension.

\section{REFERENCES}

[ASS] I. Assem, D. Simson, A. Skowroński, Elements of the representation theory of associative algebras. Vol. 1. Techniques of representation theory, London Mathematical Society Student Texts, 65, Cambridge University Press, Cambridge, 2006. MR2197389 (2006j:16020)

[AvI] L.L. Avramov, S. Iyengar, Gaps in Hochschild cohomology imply smoothness for commutative algebras, Math. Res. Lett. 12 (2005), no. 5-6, 789-804. MR2189239 (2006i:13028)

[AV-P] L.L. Avramov, M. Vigué-Poirrier, Hochschild homology criteria for smoothness, Internat. Math. Res. Notices (1992), no. 1, 17-25. MR1149001 (92m:13020)

[BM1] P.A. Bergh, D. Madsen, Hochschild homology and global dimension, Bull. Lond. Math. Soc. 41 (2009), no. 3, 473-482. MR2506831(2010e:16014) 
[BM2] P.A. Bergh, D. Madsen, Hochschild homology and split pairs, Bull. Sci. Math. 134 (2010), no. 7, 665-676. MR2725172

[BGMS] R.-O. Buchweitz, E. Green, D. Madsen, Ø. Solberg, Finite Hochschild cohomology without finite global dimension, Math. Res. Lett. 12 (2005), no. 5-6, 805-816. MR2189240 (2006k:16019)

[CaE] H. Cartan, S. Eilenberg, Homological Algebra, Princeton University Press, Princeton, NJ, 1956. MR0077480 (17:1040e)

[Cib] C. Cibils, Cyclic and Hochschild homology of 2-nilpotent algebras, K-Theory 4 (1990), no. 2, 131-141. MR.1081656 (91j:19007)

[ENN] S. Eilenberg, H. Nagao, T. Nakayama, On the dimension of modules and algebras, IV. Dimension of residue rings of hereditary rings, Nagoya Math. J. 10 (1956), 87-95. MR.0078981 (18:9e)

[Han] Y. Han, Hochschild (co)homology dimension, J. London Math. Soc. (2) 73 (2006), no. 3, 657-668. MR 2241972 (2007c:16018)

[Hap] D. Happel, Hochschild cohomology of finite-dimensional algebras, in Séminaire d'Algèbre Paul Dubreil et Marie-Paul Malliavin, 39ème Année (Paris, 1987/1988), Lecture Notes in Mathematics 1404, Springer, Berlin, 1989, 108-126. MR1035222 (91b:16012)

[Igu] K. Igusa, Notes on the no loops conjecture, J. Pure Appl. Algebra 69 (1990), 161-176. MR:1086558 (92b:16013)

[IgZ] K. Igusa, D. Zacharia, On the cyclic homology of monomial relation algebras, J. Algebra 151 (1992), 502-521. MR.1184046 (93h:19005)

[Skö] E. Sköldberg, The Hochschild homology of truncated and quadratic monomial algebras, J. London Math. Soc. (2) 59 (1999), no. 1, 76-86. MR.1688490(2000g:16015)

[SV-P] A. Solotar, M. Vigué-Poirrier, Two classes of algebras with infinite Hochschild homology, Proc. Amer. Math. Soc. 138 (2010), no. 3, 861-869. MR.2566552 (2011a:16021)

Institutt for Matematiske FAG, NTNU, N-7491 Trondheim, Norway

E-mail address: bergh@math.ntnu.no

KLMm, AMSS, Chinese Academy of Sciences, Beijing 100190, People's Republic of CHINA

E-mail address: hany@iss.ac.cn

Department of Mathematics, 215 Carnegie, Syracuse University, Syracuse, New York $13244-1150$

E-mail address: dmadsen@syr.edu

Current address: Profesjonshøgskolen, Universitetet i Nordland, 8049 Bodø, Norway

E-mail address: dag.oskar.madsen@uin.no 